\title{
AN ALGORITHM FOR PREDICTING ATTITUDES OF FLOATING BODIES WITH ARBITRARY CONFIGURATIONS IN LIQUIDS
}

\author{
Jiann-Lin Chen \\ Department of Mechanical and Automation Engineering, I-Shou University, Taiwan, R.O.C., james88@isu.edu.tw \\ Chun-I Chen \\ Department of Industrial Engineering and Management, I-Shou University, Taiwan, R.O.C \\ Li-Ming Chu \\ Department of Mechanical and Automation Engineering, I-Shou University, Taiwan, R.O.C.
}

Follow this and additional works at: https://jmstt.ntou.edu.tw/journal

Part of the Electrical and Computer Engineering Commons

\section{Recommended Citation}

Chen, Jiann-Lin; Chen, Chun-l; and Chu, Li-Ming (2010) "AN ALGORITHM FOR PREDICTING ATTITUDES OF FLOATING BODIES WITH ARBITRARY CONFIGURATIONS IN LIQUIDS," Journal of Marine Science and Technology. Vol. 18: Iss. 6, Article 11.

DOI: $10.51400 / 2709-6998.1945$

Available at: https://jmstt.ntou.edu.tw/journal/vol18/iss6/11

This Research Article is brought to you for free and open access by Journal of Marine Science and Technology. It has been accepted for inclusion in Journal of Marine Science and Technology by an authorized editor of Journal of Marine Science and Technology. 


\title{
AN ALGORITHM FOR PREDICTING ATTITUDES OF FLOATING BODIES WITH ARBITRARY CONFIGURATIONS IN LIQUIDS
}

\author{
Jiann-Lin Chen*, Chun-I Chen**, and Li-Ming Chu*
}

Key words: algorithm, floating body, attitude, submersible.

\begin{abstract}
A robust and efficient algorithm is proposed to quasiunsteadily predict attitudes of floating bodies with arbitrary configurations. Numerical techniques for accuracy and computing time saving are addressed. A floating cylinder was modeled as a benchmark, by theoretical and experimental approaches, to validate the developed program. Attitudes of two kinds of floating bodies, which are a body composed of multiple objects and a submarine-like submersible, were simulated to show the feasibility of the present algorithm. Interestingly, the simulated results of a slender body, such as a cylinder or a submersible, show that the attitude variation is very sensitive to the longitudinal movement of its center of gravity around certain region.
\end{abstract}

\section{INTRODUCTION}

Lots of problems arise when the hydrodynamics of marine vehicles are considered, but clearly a vehicle must be stable in its position when alongside a jetty or on the sea, otherwise an examination of the more complete dynamic problems is largely a fruitless exercise [8]. However, whether those belong to hydrostatic or hydrodynamic problems, there are two forces of overwhelming importance which are always present, namely weight and buoyancy. Thus, a body floating in the liquid could be inclined due to variations of its buoyancy and weight, both in magnitude and position, and the attitudes are strongly related to its floating state of equilibrium $[1,4,16]$. The attitude then becomes an issue for those floating bodies at rest or in a motion. The American research ship, Flip (FLoating Instrument Platform), is an interesting application of body floatation [11]. It was designed to float not only like a conventional surface vessel with its keel horizontal but also with

Paper submitted 11/21/08; revised 10/24/09; accepted 11/09/09. Author for correspondence: Jiann-Lin Chen (e-mail: james88@isu.edu.tw).

*Department of Mechanical and Automation Engineering, I-Shou University, Taiwan, R.O.C.

**Department of Industrial Engineering and Management, I-Shou University, Taiwan, R.O.C. its keel vertical after suitable flooding. Moreover, due to its unique ability to provide a heave-stable operating location in open ocean environments, the conceptual design for a new FLIP II has been proposed to meet current and future scientific mission requirements [14]. In fact, the buoyancy and weight of a heavy body are not only relevant to its stability of floatation, but also to its deploying and retrieving problems resulting from its floating attitudes in sea trials. Empirically, Chen and $\mathrm{Wu}[6]$ proposed a correlation to acquire the floating attitudes by trimming the center of gravity (CG) of an underwater vehicle while retrieved after sea trials. However, not many researches focused on study for attitudes of floating vehicles and this is the motivation of this research to develop a computing algorithm to study it. The attitudes of a floating cylinder with uniform material were investigated by Dugdale [10]. It was shown that a cylinder having a length less than 0.707 of its diameter will float with its axis vertical while the length exceeding its diameter will float with its axis horizontal. Chen et al. [7] investigated the relations of relevant dimensionless parameters, such as the inclination angle, weight magnitude and location of CG for a floating cylinder. In addition, the measurement of physical properties of a general body, e.g. the floating attitude and the position of CG, is not an easy task. Buyanov [3] proposed a method and device for determining the CG by taking advantage of the test body floating in a tank, but the device was somewhat complicated and not suitable for heavy vehicles such as torpedoes and submarines. Nowadays, by virtue of powerful computer hardware and software, the technique of computer-aided engineering (CAE) prevails and can be applied to obtain the properties of floating bodies, whether in design stage or in test and evaluation one [15]. In the past decades, the computational fluid dynamics (CFD), one of CAE, has been employed to successfully solve plenty of fluid problems. For CFD approach, the governing equations from the fluid dynamics in physical domain are reformulated in computational domain and then discretized to form a calculation procedure [13]. Furthermore, the quality of mesh distribution as well as the proper interpolation play a key role in its numerical accuracy $[2,12]$.

The motivation of this study came from the deploying and retrieving problems of an exercise submersible in sea trials [6]. That submersible was a prototype of a heavy torpedo in its 
configuration of body of revolution. When it floated after the first test run, it was oriented nearly 70 degrees vertically in the sea, which caused a lot of trouble in deploying and retrieving. Meanwhile, rapidly acquiring the CG during the weight and balance procedure before sea trials was a big concern because of the huge resources involved. In other words, a tool to predict the inclinations caused by weights and their locations is necessary in the design stage as well as during testing and evaluation. One other engineering application may be for submarines surfacing during missions. The attitudes of a flooding submersible are strongly related to the buoyancy-center shift longitudinally rather than in lateral direction. A basic issue in the design of a submarine is the provision of high strength bulkheads to isolate selected parts of the hull and so limit the extent of flooding throughout the hull in the event of an accident. The present proposed algorithm could be employed to simulate a sinking submarine and indicate the preliminary concept for escape policy in the early stages of this submarine design. Moreover, it is possible to simulate the water tank experiment for body floatation by a numerical model so that numerical experiments can be performed without a real tank, and the body's properties can be systematically changed for parametric studies. The investigation of the attitude of a cylinder related to some parameters in our previous work implies that the data bank can be established without difficulty by using the present algorithm for floating vehicles with complex configurations [7].

The present approach employs the numerical techniques from CFD in constructing an algorithm but in more straightforward way based on our previous work [8]. In this study, the first step is to acquire the attitudes of a floating cylinder, as a benchmark, for some specific cases theoretically, experimentally and numerically. Thereafter, the algorithm of the developed program for bodies with arbitrary configurations is introduced. The attitudes of several bodies composed of multiple objects, including a submarine-like submersible, are demonstrated via a human-machine interface. Finally, some interesting conclusions are addressed according to the simulated results.

\section{MODELLING}

\section{A General Physical Model}

For problems of body floatations corresponding to statical stability, the body settles until the buoyant force equals the weight and rotates until the centers of buoyancy and gravity are in perfect vertical alignment. In this study, we examine the forces involved as the body is disturbed from equilibrium and the disturbance is assumed to cause a small rotation of the body about a relevant axis. During our analysis, statical stability means the tendency of the body to return to its initial equilibrium position, in which with only the direction and magnitude of moments are of concern and velocity of rotation and inertial effects are ignored [16]. Figure 1 shows the gen-

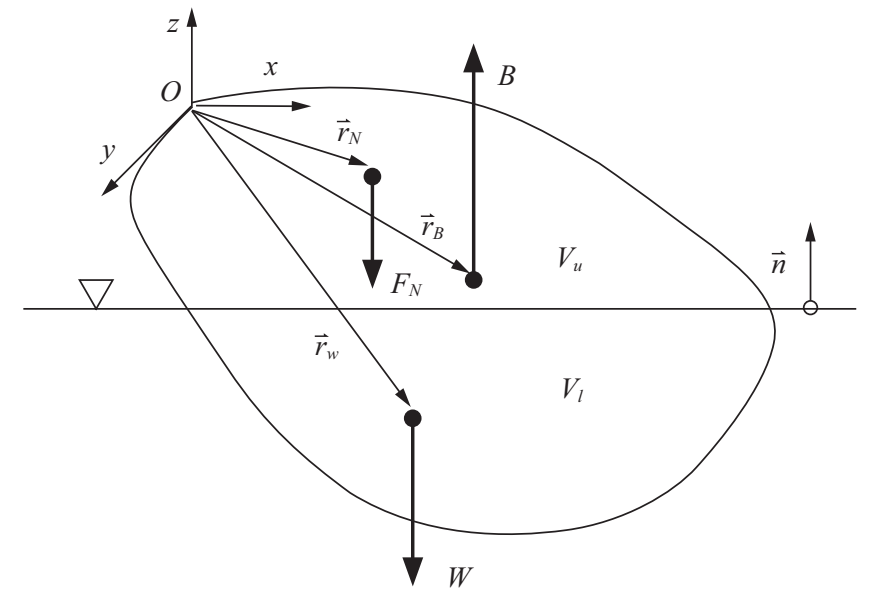

Fig. 1. A general description of a floating body.

eral description of a floating body at rest in a liquid with the origin of the coordinate system $O x y z$ fixed arbitrarily with respect to the Earth. The $\vec{n}$ is the normal vector on the liquid plane, $V_{u}$ represents the volume above the liquid plane and $V_{l}$ is the immersed volume. Accordingly, the buoyant force exerted by the air on $V_{u}$ is ignored as compared with one by the liquid on $V_{l}$. It is noted that lumped system is introduced in the present analysis, where $\mathbf{B}$ stands for the total buoyant force exerted by the liquid as the whole body submerges and $\mathbf{W}$ stands for the gross weight of the body. The conditions for a specific attitude can be stated from fundamental identities of static mechanics. Thus, we have the following expression:

$$
\sum \vec{F}=\vec{F}_{B}+\vec{F}_{W}+\vec{F}_{N}=(B-W) \vec{k}+\vec{F}_{N}=0
$$

where the virtual total buoyant force is $\vec{F}_{B}=B \vec{k}$, the weight is $\vec{F}_{W}=-W \vec{k}$. Based on these, the relation follows as $\vec{F}_{N}=$ $-(B-W) \vec{k}=f\left(V_{u}\right)$, where $\vec{F}_{N}$ indicates a negative buoyant force because the body is partly submerged.

Besides, the moment balance becomes

$$
\sum \vec{M}=\sum \vec{r} \times \vec{F}=\vec{r}_{B} \times \vec{F}_{B}+\vec{r}_{W} \times \vec{F}_{W}+\vec{r}_{N} \times \vec{F}_{N}=0
$$

where $\vec{r}_{B}, \vec{r}_{W}$ and $\vec{r}_{N}$ are the position vectors of $\vec{F}_{B}, \vec{F}_{W}$ and $\vec{F}_{N}$ respectively.

Obviously, the volume above the liquid $V_{u}$ is related to the body's configuration and indirectly related to its properties, such as the total buoyancy and weight as well as their locations. Equations (1) and (2) confirm that the buoyant force is equal to the weight force and the CG is in the same line with the center of buoyancy (CB) vertically.

\section{A Cylinder Model}

Following the previous description, the formula governing the attitudes of a floating cylinder has been derived [7]. This cylinder case with theoretical and experimental data is used as 


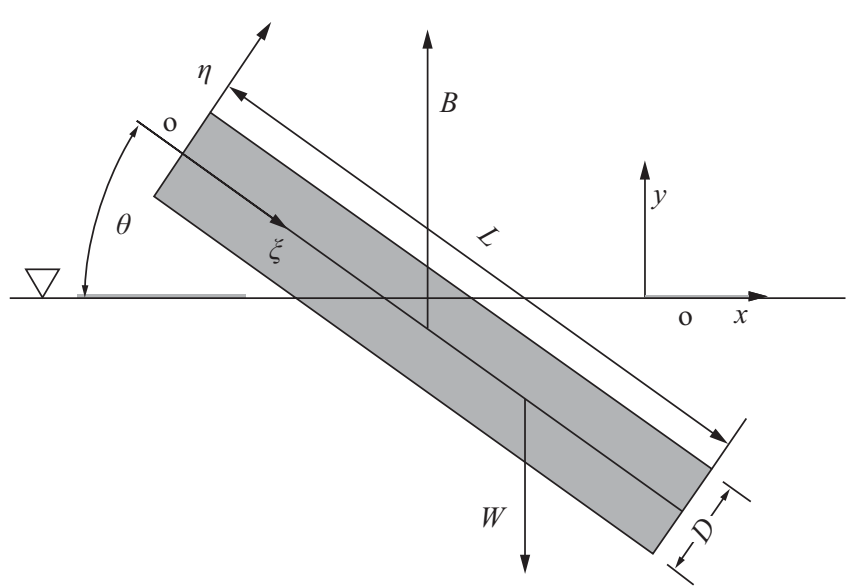

Fig. 2. Physical model of a floating cylinder.

a benchmark to validate the proposed algorithm. Figure 2 shows the sketch of the floating cylinder at rest in a liquid, with diameter $D$, length $L$ and other notations. Besides, the $\mathrm{CG}$ is located at the position with coordinates, $\left(\xi_{W}, \eta_{W}\right)$. Based on the calculus and hydrostatics, the governing formula takes the form [7]:

$$
\Theta^{3}+\left(16 \omega^{2}-32\left(L / D-\xi_{W}^{\prime}\right)+2\right) \Theta=-32 \eta_{W}^{\prime} \omega
$$

where $\Theta=\cot \theta, \xi_{W}^{\prime}=\xi_{W} / D, \eta_{W}^{\prime}=\eta_{W} / D$. The $\omega$ is defined as the weighting of gravity force $W / \tau$, and $\tau$ is equal to $\pi D^{3} \gamma_{f} / 4$, where $\gamma_{f}$ indicates the specific weight of the present liquid. Accordingly, the constraints for valid parameters, such as $-0.5<$ $\eta^{\prime}<0$ and $1<\omega<L / D$ are prescribed due to the simplified physical model. Equation (3) clearly shows that attitude angle $\theta$ is a function of dimensionless terms $\xi_{W}^{\prime}, \eta_{w}^{\prime}$ and $\omega$, which represent the longitudinal location of the $\mathrm{CG}$ from the top end, the biased location of the $\mathrm{CG}$ in radial direction as well as the weight magnitude respectively. Root-finding schemes were used to find the roots of (3) when it was expressed in a polynomial form, $f(x)=0$.

The associated experiment was carried out in a water tank. The cylinder model was made of an acrylic plastic tube, which was transparent so that the CG of ballasts could be determined by processing the digital pictures via the AutoCAD software. Two steel balls were used for the ballasts, then the experiments were conducted by putting those steel balls inside the tube with a small piece of styrofoam to adjust their positions. When the cylinder was floating in an equilibrium situation, digital pictures were taken. Then the attitude angles were calculated by uploading pictures to the computer for further image processing. Figure 3 shows experimental data compared with one of the theoretical results from (3). The attitudes $\theta$ were obtained theoretically and numerically as the position of the CG varied with the given parameters, $\omega=3.5145, L / D=7.9227$, $\eta_{w}^{\prime}=-0.11622$. Overall speaking, the tendency of the comparison shows satisfactory. It indicates that it was pretty

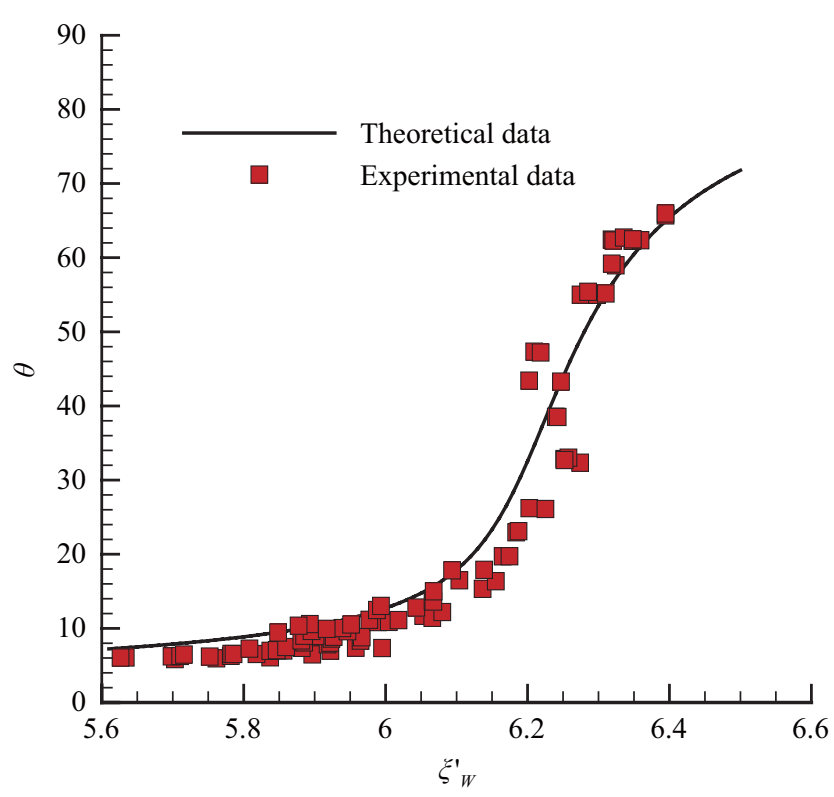

Fig. 3. Comparisons of experimental data and theory.

difficult to accurately measure the location of CG of a body, especially in radial direction. Obviously, minor errors could cause significant deviation between theoretical and experimental data in the region with steeper slope. To remedy it, the present numerical approach has been employed to obtain the attitudes of bodies with arbitrary configurations.

\section{NUMERICAL SIMULATIONS}

The Microsoft Visual Basic language (VB) was adopted to write the present code because the VB is not only a programming language, but also a complete graphical development environment [4]. This environment allows users with little programming experience to develop friendly human-machine interfaces, which are useful in parametric studies [8]. Also, it is an Object-oriented programming language with supports of lots of resource, such as ActiveX controls and third party programs.

\section{A Proposed Algorithm, BDM}

For vehicles other than regular configurations, a useful program has been developed to simulate their attitudes. The present numerical algorithm, BDM (Body Decomposition Method), is motivated by the lumped-system concept from conventional naval architecture [9]. A body with general configuration can be decomposed to small finite elements numerically. It means that a vehicle simulated by the BDM can be constructed by $3 \mathrm{D}$ meshes; thereafter, the vehicle's configuration can be accurately described when meshes approach infinitesimally small. This mesh approach is also very similar to grid distribution in traditional finite volume method [12]. In the present work, a body is constructed to be composed of objects with regular configurations such as sphere, cylinder, cuboid, cone, disk as 


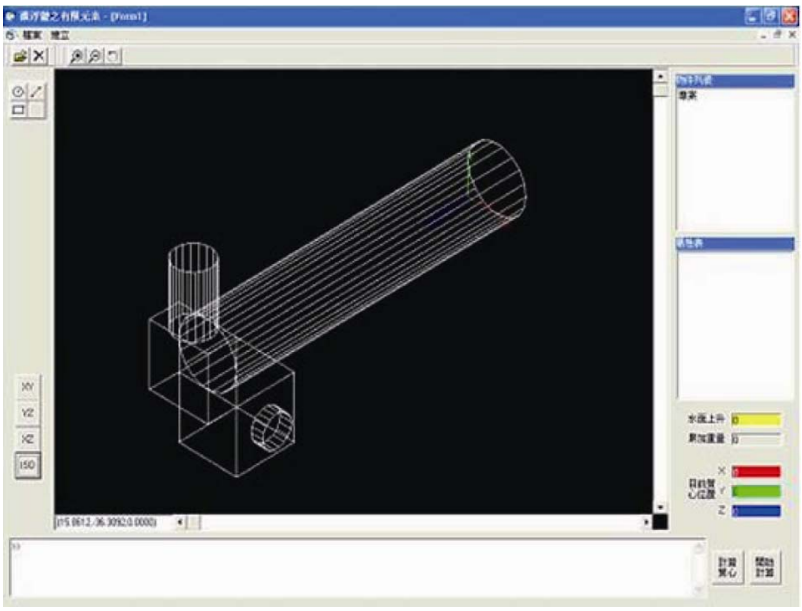

Fig. 4. A human-machine interface by the VB language.

well as taper, which can be easily defined by mathematical expressions for simulation. For instance, Fig. 4 shows that a body with five objects in a window, which is a user-friendly interface by the VB language. Similarly, any arbitrary body can be constructed numerically by connecting various individual objects before executing the present program. Thereafter, by ignoring bodies' velocity of rotation and inertial effects, the computing procedure of the proposed algorithm can be applied to acquire the attitudes of floating bodies quasi-unsteadily. As the calculation is performed, the animation of liquid surface flooding the body can be made and displayed on the screen simultaneously.

The simulation steps of the BDM are stated below and Fig. 5 illustrates the relevant notations:

(a) The $X Y Z$ coordinates, with the origin at upper surface corner, are fixed in the virtual computational domain.

(b) The body coordinates $\xi \eta \gamma$ are fixed in a body of interest; this coordinates can be transformed to $X Y Z$ without difficulty.

(c) Define $N$ objects $O_{n}$ of the body, $\left\{O_{n}, n=1 . . N\right\}$. Input the geometric configuration denoted by volumes $V_{n},\left\{V_{n}, n=\right.$ $1 . . N\}$ and the position for each object. Give the specific weight $\gamma_{n}$ of each object, $\left\{\gamma_{n}, n=1 . . N\right\}$, and give the specific weight of the liquid, $\gamma_{l}$.

(d) Determine the computational domain by a rectangular parallelepiped, with length $\left(L_{X}, L_{Y}, L_{Z}\right)$, covering the body by linking each object as a whole. The program then automatically calculates the following body's gross weight

$$
W=\sum_{N=1}^{N} \gamma_{n} V_{n}
$$

The location of CG in the $X$ direction is expressed as

$$
X_{C W}=\sum_{n=1}^{N} \gamma_{n} V_{n}\left(X_{n}\right)_{C G} / W
$$

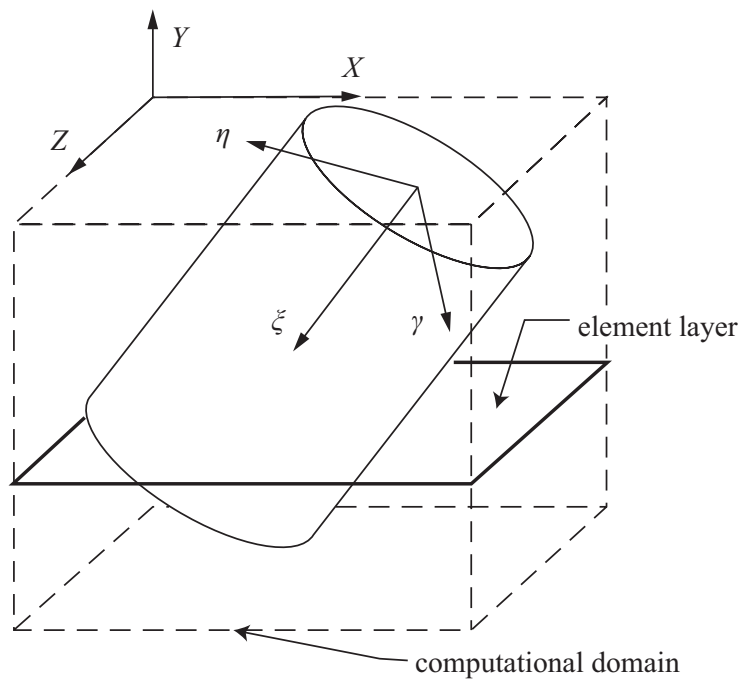

Fig. 5. Notations for an element layer in computational domain.

and similarly for the $Y, Z$ directions.

(e) Divide the domain into small elements, layer by layer in the $X$-Z plane inside $\left(L_{X}, L_{Y}, L_{Z}\right)$, from the bottom to satisfy accuracy requirement. A layer is constructed by $I \times K$ elements, with $i=1 . . I$ and $k=1 . . K$.

(f) Flood the body from below along the $Y$ axis, from layer 1 to $j$ with maximum $J$ to simulate the liquid surface in the $X-Z$ plane.

(g) As calculation proceeds, sum the elements under the liquid surface and calculate the relevant buoyant force as

$$
\tilde{B}=\sum_{j=1}^{j}\left(\sum_{n=1}^{n} \sum_{k=1}^{k} \sum_{i=1}^{i}\left(V_{n}\right)_{i, k} \gamma_{n}\right)_{j}
$$

The location of CB in the $X$ direction is expressed as

$$
X_{C B}=\sum_{j=1}^{j}\left(\sum_{n=1}^{n} \sum_{k=1}^{k} \sum_{i=1}^{i}\left(V_{n}\right)_{i, k} \gamma_{n}\left(X_{n}\right)_{C B}\right)_{j} / \tilde{B}
$$

and similarly for the $Y, Z$ directions.

(h) Stop flooding the body when the calculated buoyant force equals to its weight, that is, $\tilde{B}=W$.

(i) Judge if the net moment about the $Z$ axis, $M_{Z}=\tilde{B} x_{C B}-$ $W x_{C G}$, and the $X$ axis, $M_{X}=\tilde{B} z_{C B}-W z_{C G}$, are approaching zero and within the required error bound individually. If yes, the calculation is terminated and the attitude of the body is determined.

(j) If not, rotate the body about the $X$ axis and/or the $Z$ axis incrementally by small angles and recheck $\tilde{B}=W$ and the net moment. Note that the increment may overshoot; it is better to reduce the rotating angle gradually as the calculated net moment approaches to null.

The calculation steps of the BDM are also expressed by 


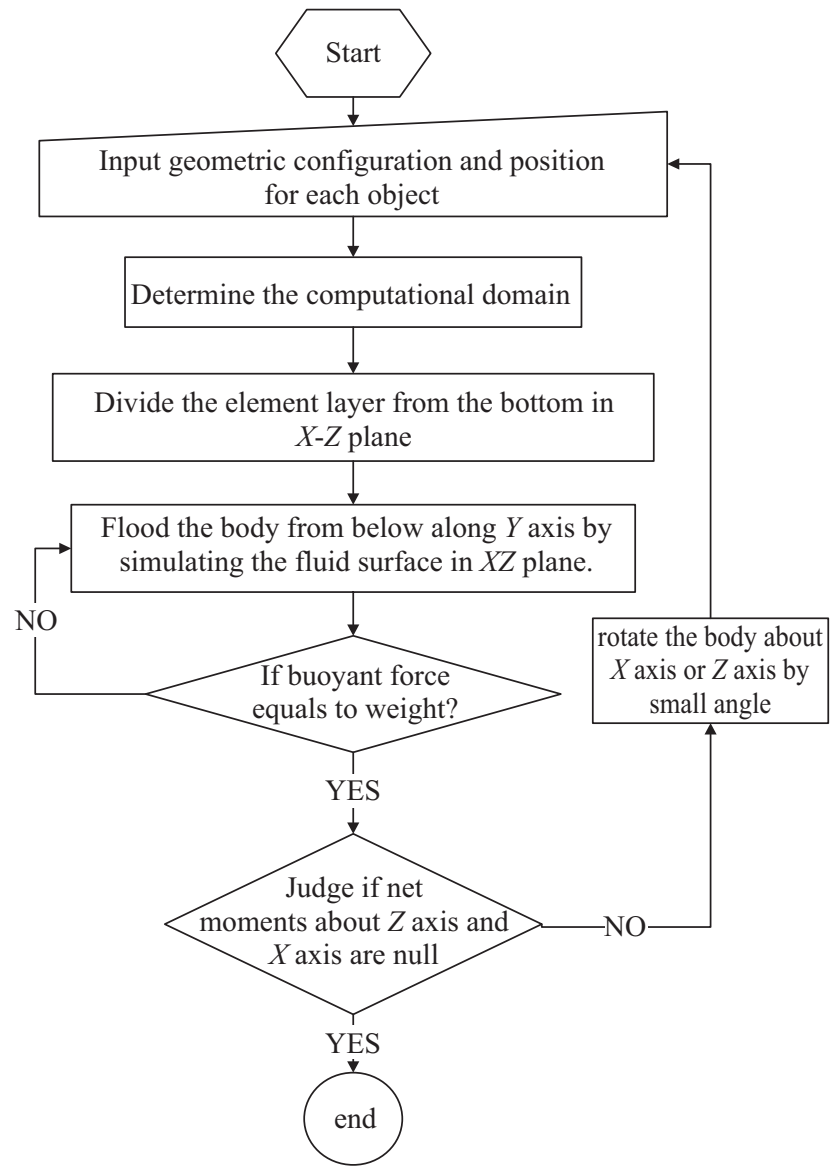

Fig. 6. The flowchart of the BDM algorithm.

flowchart in Fig. 6. This algorithm has been proved robust and efficient. In reality, the present numerical technique is somewhat similar to the finite volume method, but the tedious mathematical formulation and matrix calculation are avoided. Thus, case studies can be performed in ordinary desktop computers by virtue of the following techniques.

\section{Techniques for Computing}

As the element size becomes smaller or the body size become bigger, the number of elements for the computational domain becomes larger. Theoretically, the computing time is approximately exponentially proportional to the product of respective element number of three sides of the computational domain. For instance, it takes eight-fold time when 0.1 unit mesh size is adopted instead of 0.2 unit for computation. Fortunately, we calculated the properties layer by layer according to the present quasi-unsteady algorithm. It means that memory for computation is needed merely for elements of the relevant layer. In addition, the object Timer control in VB was employed to save memory if fine elements were used. Timer allows us to set a time interval to execute an event after that interval continuously. It is useful when we want to execute certain applications after a certain interval. Say we want to create a backup of our data processing in every minute. We

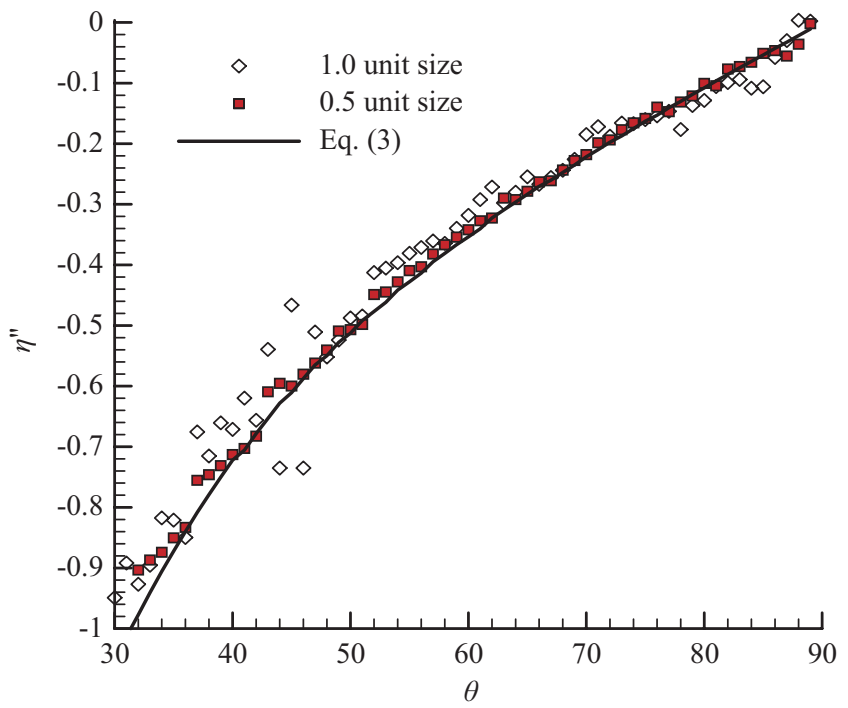

Fig. 7. Element size effect upon numerical accuracy.

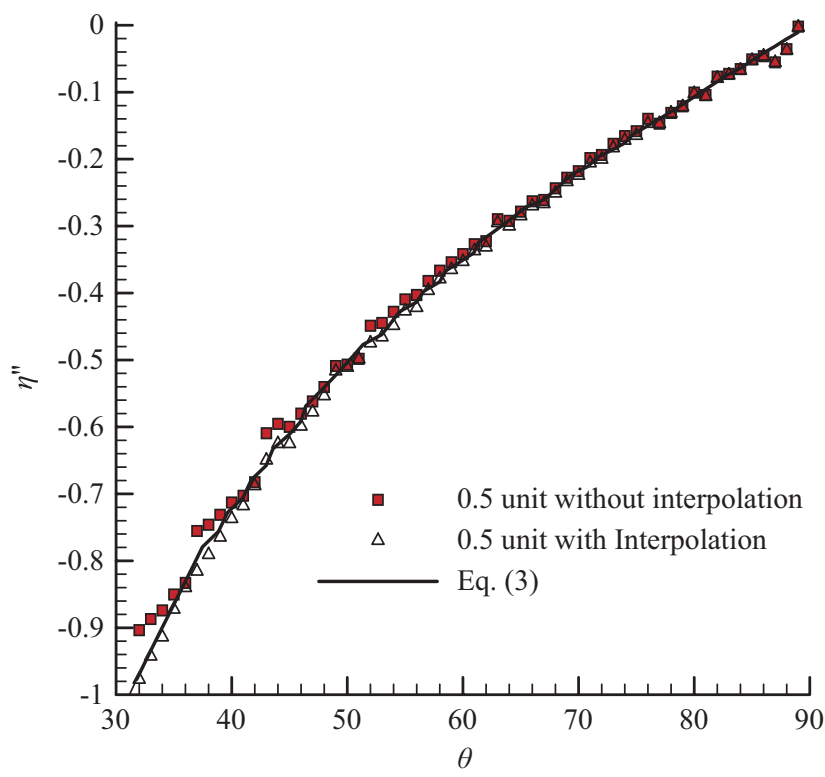

Fig. 8. Interpolation effect upon numerical accuracy.

can make a routine which will take the backup and call that routine on Timer's event and set timer interval for a minute. Thus, the animation clip can be made from the backup files event by event. The default setting, Timer(1), in VB corresponds to 0.001 second executing time for an interval.

In order to know the relationship between element size and accuracy, comparisons for a specific case with/without interpolation are demonstrated in Figs. 7 and 8. The case was about a cylinder with 800 unit weights, height 100 unit lengths and diameter 10 unit lengths. The simulation steps were similar to the previous description. However, the attitudes of this floating cylinder were set fixed during calculation, which are indicated by $\theta$ in the horizontal axis; the corresponding centers 


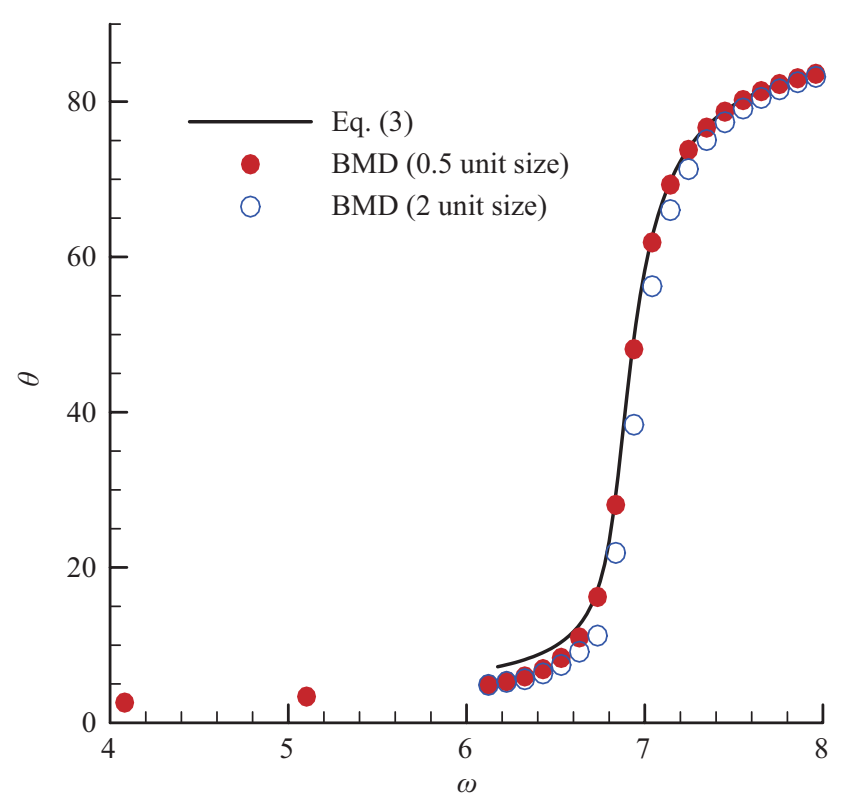

Fig. 9. Comparisons of theory and the BDM algorithm.

of gravity in radial location were then computed and indicated by $\eta^{\prime \prime}$ in the vertical axis with $\eta^{\prime \prime}=\eta_{W} /(D / 2)$. Figure 7 illustrates that the data of 1 unit element size appear obviously deviated from the theoretical solutions. In this case, three sizes, $1,0.5$ and 0.1 have been tested; the results of 0.1 were nearly coincided with the theory and not shown here. In order to further reduce the discrepancy and save the memory and CPU time, the interpolating technique was employed to deal with the boundary surface in $X-Z$ plane when the element layer was not coincided with the liquid surface while flooding, which was leveled up till body's weight equal to the buoyancy. Although the interpolation was linear, the results show it is effective as illustrated in Fig. 8. Thus the element of 0.5 unit length associated with linear interpolation was adopted in the case studies.

Comparisons of other cases to show the robustness and accuracy of the present algorithm are illustrated in Fig. 9. Figure 9 shows the attitudes from theory and present simulated results with parameters, $L / D=8, \xi_{W}^{\prime}=4.6$ and $\eta_{w}^{\prime}=-0.065$, of the floating cylinder for various dimensionless weights. In this simulation, the centers of gravity were biased by putting some elements with different densities within the cylinder. Herein the densities of those computed elements were changed proportionally to vary the magnitudes of weight, thus the biased CG could be kept invariant. Three mesh systems, 0.5, 1.0 and 2.0 unit lengths, were employed in calculations with proper interpolations. However, only the results from mesh systems, 0.5 and 2.0 units, are illustrated in Fig. 9 because the lines from 0.5 and 1.0 units are almost indistinguishable. As described previously, the computing time for case 0.5 unit was eight-fold than that of 1.0 unit. Without loss of accuracy, the 0.5 unit mesh system was used in model tests. Also note that the compared results of theory and present algorithm with 0.5

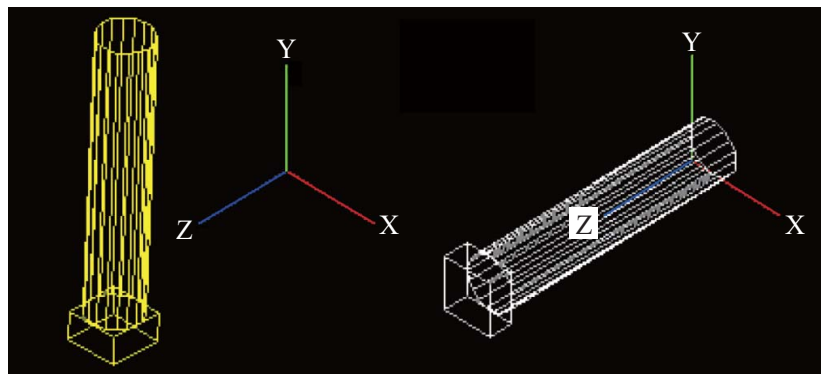

Fig. 10. A body composed of one cylinder and one cuboid.

unit mesh system are satisfactory except for $\omega$ less than about 6.8. The reason is that the water surface has flooded the top disk of the cylinder and the theoretical solution failed in such case. In other word, the results from theory and present algorithm for $\omega$ less than about 6.8 in Fig. 9 indicate different cases. Furthermore, the simulations can be extended to cases of small $\omega$ values, which represent cases of the cylinder floating nearly horizontally. Thus, one can learn from Fig. 9 that the present BDM algorithm is robust in simulating attitudes of bodies floating in liquids.

\section{MODEL TESTS AND DISCUSSIONS}

Two typical models were used to demonstrate the present algorithm. The desktop computer with PIII 3.2 G Hz CPU and 1 GB RAM was used to do the case study. The elapsed time to execute the case of 7200 meshes with 0.1 unit was around 6.5 minutes. In reality, the total computing time was associated with the initial position of the body as we started to perform the calculation. With several trials for the initial position, the CPU time could be reduced dramatically. Technically, employing the parallel computing with a multi-processor computer for execution will significantly enhance the performance efficiency. Besides, the simulation steps for model tests in this work were animated quasi-unsteadily by the present friendly human-machine interface.

\section{Bodies with Arbitrary Configurations}

Figure 10 shows the attitude of a simple body floating in water, which is composed of two objects, one cylinder and one cuboid. The body on the right-hand side in Fig. 10 is in its initial position before the simulation starts. After completing computation, the body appears vertical and becomes statically stable as shown on the left-hand side. This computing procedure is very straightforward and quasi-unsteady in seeking for the balances of moments and forces concurrently. Similarly, more cases have been demonstrated and the relevant characteristics are tabulated in Tables 1 and 2. The dimensions (in meter) and specific gravity for each object for cases 1 to 5 are tabulated in Table 1, where the coordinate set for a cylinder indicates (length, diameter, specific gravity) and for a cuboid indicates (length, breadth, height, specific gravity). Consequently, the centers of gravity and buoyancy as well as the 
Table 1. Geometry and specific gravity for a body (in meter).

\begin{tabular}{|l|l|l|l|l|}
\hline & \multicolumn{3}{|c|}{ Objects of a Body } \\
\hline & \multicolumn{2}{|c|}{ Cylinder(s) } & \multicolumn{2}{|c|}{ Cuboid(s) } \\
\hline Case 1 & \multicolumn{2}{|c|}{$(50,10,0.2)$} & \multicolumn{2}{c|}{$(10,10,5,3)$} \\
\hline Case 2 & \multicolumn{2}{|c|}{$(50,10,0.2)$} & \multicolumn{2}{c|}{$(10,10,5,2)$} \\
\hline Case 3 & \multicolumn{2}{|c|}{$(50,10,0.2)$} & \multicolumn{2}{c|}{$(20,10,5,2)$} \\
\hline Case 4 & 1 & $(50,10,0.2)$ & 1 & $(10,10,5,3)$ \\
\cline { 2 - 5 } & 2 & $(2,5,0.2)$ & 2 & $(10,10,10,0.2)$ \\
\cline { 2 - 5 } & 3 & $(10,6,0.8)$ & & \\
\hline \multirow{3}{*}{ Case 5 } & 1 & $(50,10,0.2)$ & 1 & $(10,10,5,2)$ \\
\cline { 2 - 5 } & 2 & $(2,5,2)$ & 2 & $(10,10,10,2)$ \\
\cline { 2 - 5 } & 3 & $(10,6,0.8)$ & & \\
\hline
\end{tabular}

Table 2. Characteristics of bodies with multiple objects (in meter).

\begin{tabular}{|c|l|l|l|}
\hline Case & Locations of CG & Locations of CB & Attitude $(X, Z)$ \\
\hline 1 & $(5,5,43.05)$ & $(5,6.18,43.04)$ & $\left(87^{\circ}, 0^{\circ}\right)$ \\
\hline 2 & $(5,5,40.40)$ & $(5,3.39,40.4)$ & $\left(10.5^{\circ}, 0^{\circ}\right)$ \\
\hline 3 & $(8.59,5,44.8)$ & $(8.6,7.04,44.7)$ & $\left(91.9^{\circ}, 138.5^{\circ}\right)$ \\
\hline 4 & $(5.96,6.0,43)$ & $(5.96,0.36,43)$ & $\left(2.13^{\circ}, 75.5^{\circ}\right)$ \\
\hline 5 & $(10.2,5.55,48.5)$ & $(10.2,11,48.5)$ & $\left(117.8^{\circ}, 92.2^{\circ}\right)$ \\
\hline
\end{tabular}

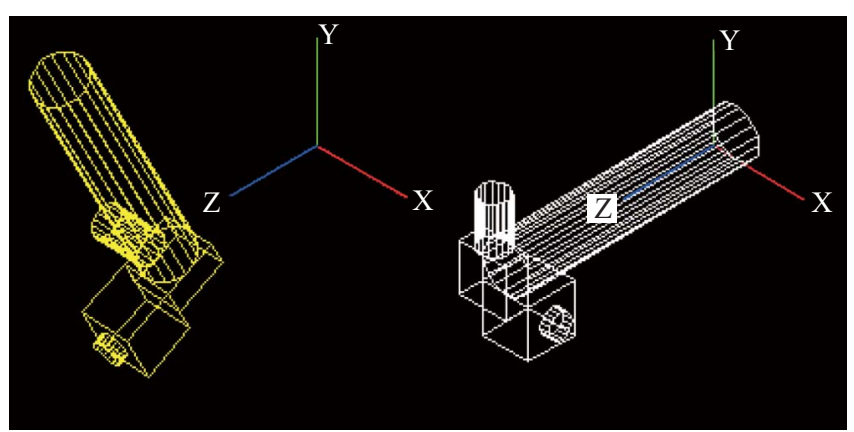

Fig. 11. A body composed of three cylinders and two cuboids.

floating attitudes are expressed in sets of coordinates in Table 2. Note that the first argument of last column in Table 2 means the rotating angle about the $X$ axis; the second one indicates the rotating angle about the $Z$ axis. Interestingly, although the $\mathrm{CG}$ is above the $\mathrm{CB}$ in cases 1 and 4 , the bodies in these two cases still appear statically stable. We couldn't get convergent solutions when both bodies were set upside down. Moreover, either case 4 or 5 shows the attitude of the body with multiple objects, which contain three cylinders and 2 cuboids. In case 5 , which is also demonstrated schematically in Fig. 11, the body was initially set in horizontal position; after quasi-unsteadily completing the simulation, the body would rotate at $92.2^{\circ}$ roll angle and at $117.8^{\circ}$ pitch angle to reach to the final state. Generally, the elapsed time for simulating the body with two objects was less than 5 minutes, while for the body with 5 objects less than 15 minutes. As described in the previous
Table 3. Physical characteristics of the submersible.

\begin{tabular}{|l|l|c|}
\hline \multicolumn{1}{|c|}{ Objects } & \multicolumn{1}{c|}{ Dimensions (in meter) } & Specific gravity \\
\hline Half sphere & $D=20, t=0.4$ & 7.8 \\
\hline Cylinder & $D=20, L=160, t=0.4$ & 7.8 \\
\hline Cone & $D_{1}=20, D_{2}=4.5, t=0.4$ & 7.8 \\
\hline Sail & $L=8, w=16, H=10$ & 0.8 \\
\hline Disk & $D=14, t=1.5$ & 7.8 \\
\hline 4 fins at tail & $L=6, w=2, H=9$ & 0.9 \\
\hline 2 fins at tail & $L=8, w=2, H=10$ & 0.8 \\
\hline
\end{tabular}

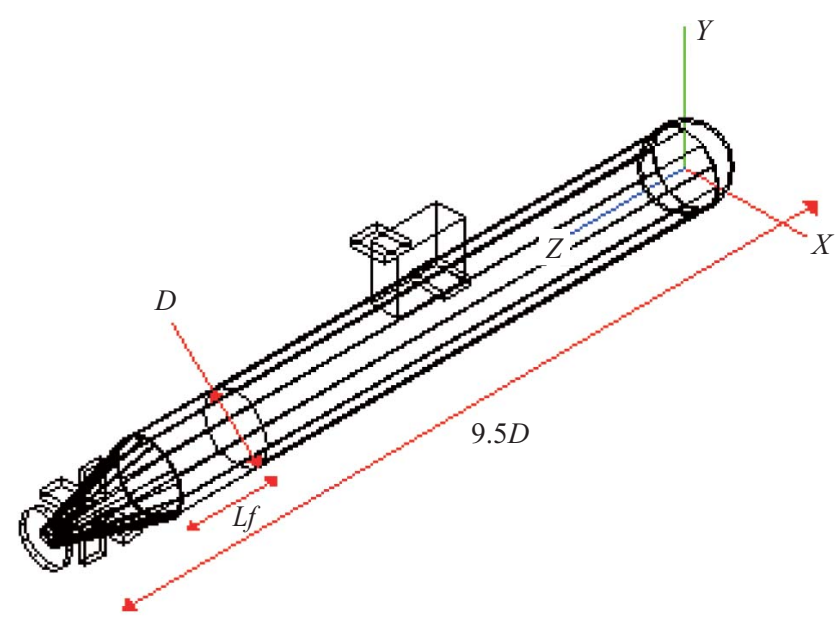

Fig. 12. The submersible model of a submarine.

sections, nowadays it will not be a problem to tackle those cases with more complicated situations if the computer hardware is upgraded accordingly.

\section{Attitudes of a Submersible}

The configuration of a submarine-like submersible in Fig. 12 was constructed to show the feasibility of the present algorithm for more practical applications. Figure 12 demonstrates the outline of the mesh system for the slender submersible with the propeller modeled by a disk and the length over diameter being 9.5. The physical characteristics are tabulated in Table 3, where $D, t, w, L, H$ indicate the diameter, thickness, breadth, length and height respectively. Figure 13 illustrates the relations of floating attitudes, $\theta$, versus the locations of $\mathrm{CG}$ in the longitudinal direction, $\xi / D$, with the different locations of $\mathrm{CG}$ in the transverse direction, $\eta / D$. Similarly, $\xi$ is the distance measured from the head of the submersible, while $\eta$ from the centerline of the submersible. Approximately, the elapsed time for simulating an attitude of the submersible was less than 25 minutes for a medium-size mesh system. The closer to 45 degree the attitude angle of the submersible appeared, the more memory and CPU time the computing consumed because of the larger range of computational domain. Interestingly, the attitudes rise dramatically around some critical range in the horizontal axis for each $\eta / D$ 


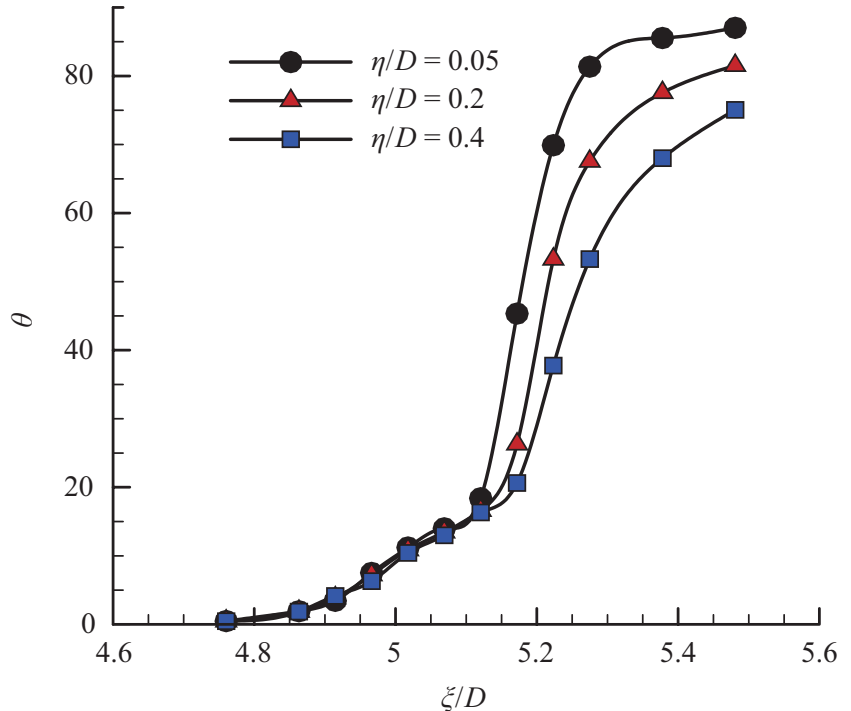

Fig. 13. Attitudes versus $\xi / D$ for different $\eta / D$.

as shown in Fig. 13. Apparently, the attitude variation is more sensitive as the CG moving along the longitudinal direction than moving along the transverse direction. Figure 13 also reveals that the submersible appears nearly level as the CG close to the middle of it regardless of the $\mathrm{CG}$ being biased in the transverse direction. Moreover, the submersible floats nearly vertical as the CG moves close to the tail of it. In fact, these phenomena are valid for slender bodies and have been revealed by (3).

\section{CONCLUSIONS}

This work presents an algorithm upon predicting attitudes of floating bodies with arbitrary configurations in liquids. Based on the results, several conclusions are drawn as follows:

1. For numerical validation, the attitudes of a floating cylinder have been obtained by theory, experiment and present numerical simulation. There are constraints while using theoretical modeling. Nevertheless, the present numerical techniques can be applied to cases with more extensive conditions.

2. The numerical techniques, such as suitable mesh size and interpolation, are demonstrated to be effective upon increasing numerical accuracy and saving computing time.

3. The cases of floating bodies composed of multiple objects were used to show the feasibility of the present simulation with the user-friendly interface. The results reveal the ro- bustness and efficiency of the present algorithm to simulate floating bodies with arbitrary configurations quasiunsteadily.

4. For a floating body with slender configuration, such as a cylinder or a submersible, the attitude variation is very sensitive to the longitudinal movement of its center of gravity around certain region.

\section{REFERENCES}

1. Allmendinger, E. E., Vergne, M. D. L., and Jackson, H., "Hydromechanical principles," Submersible Vehicle Systems Design, Society of Naval Architects and Marine Engineers, NJ, pp. 191-269 (1990).

2. Braaten, M. and Shyy, W., "A study of recirculating flow computation using body-fitted coordinates: Consistency aspects and mesh skewness," Numerical Heat Transfer, Vol. 9, pp. 559-574 (1986).

3. Bradley, J. C. and Millspaugh, A. C., Programming in Visual Basic 6.0, McGraw Hill, Boston, Ch. 12 (2004).

4. Burcher, R. and Rydill, L., Concepts in Submarine Design, Cambridge University Press, Cambridge, UK, Ch. 3 (1999).

5. Buyanov, E. V., "A method and device for determining a center of gravity," Linear and Angular Measurements, Plenum Publishing Corporation, NY, pp. 919-922 (1993).

6. Chen, J. L. and Chen, C. I., "The modeling and numerical solution for the inclination of a floating cylinder on a liquid surface," Journal of the Chinese Society of Mechanical Engineers, Vol. 27, No. 2, pp. 241-248 (2006).

7. Chen, J. L., Wu, C. H., and Hsu, C. C., "The modeling and simulation for attitudes of floating bodies with arbitrary configurations," Proceedings of 2005 Spring Simulation Multiconference, San Diego, CA, USA, pp. 179185 (2005).

8. Chen, J. L. and Wu, M. T., "The study of the attitude of a slender body floating on the sea via computer aided design," (in Chinese), Proceeding of the 25th Conference on Theoretical and Applied Mechanics (STAM), Taichung, Taiwan, pp. 222-223 (2001).

9. Clayton, B. R. and Bishop, R. E. D., Mechanics of Marine Vehicles, E. \& F. N. Spon Ltd., London, pp. 57-88 (1982).

10. Dugdale, D. S., "Stability of a floating cylinder," International Journal of Engineering Science, Vol. 42, pp. 691-698 (2004).

11. Fisher, F. H. and Spiess, F. N., "FLIP - Floating Instrument Platform," Journal of the Acoustical Society of America, Vol. 35, pp. 1633-1644 (1963).

12. Jeng, Y. N. and Chen, J. L., "Truncation error analysis of the finite volume method for a model steady convective equation," Journal of Computational Physics, Vol. 100, pp. 64-72 (1992).

13. Karki, K. C. and Patankar, S. V., "Calculation procedure for viscous incompressible flows in complex geometries," Numerical Heat Transfer, Vol. 14, pp. 295-307 (1988).

14. Laible, D. H., "FLIP II - Concept Designs to Meet Future Scientific Mission Requirements," Eos, Transactions, American Geophysical Union, Fall Meeting supplement, Abstract OS61B-0224, California (2002).

15. Lee, K., Principles of CAD/CAM/CAE System, Addison-Wesley, California, Ch. 8 (1999).

16. Zubaly, R. B., Applied Naval Architecture, Cornell Maritime Press, Maryland, USA, pp. 133-145 (1996). 